\title{
Impact of concomitant nontuberculous mycobacteria and Pseudomonas aeruginosa isolates in non-cystic fibrosis bronchiectasis
}

This article was published in the following Dove Press journal: Infection and Drug Resistance

\author{
Meng-Heng Hsieh ${ }^{1,2, *}$ \\ Chun-Yu Lin ${ }^{1-3, *}$ \\ Chen-Yu Wang ${ }^{2}$ \\ Yueh-Fu Fang ${ }^{1-3}$ \\ Yu-Lun Lo ${ }^{1,2}$ \\ Shu-Min Lin ${ }^{1,2}$ \\ Horng-Chyuan Lin ${ }^{1,2}$
}

'Department of Thoracic Medicine, Chang Gung Memorial Hospital at Linkou, Taoyuan, Taiwan; ${ }^{2}$ Department of Medicine, College of Medicine, Chang Gung University, Taoyuan, Taiwan; ${ }^{3}$ Department of Pulmonary and Critical Care, Saint Paul's Hospital, Taoyuan, Taiwan

*These authors contributed equally to this work

Correspondence: Horng-Chyuan Lin Department of Thoracic Medicine, Chang Gung Memorial Hospital at Linkou, 5 Fu-Hsing Street, Kweishan, Taoyuan 33305, Taiwan

Tel $+8863328 I 200$ ext.8470

Fax +88633287787

Email lin53424@gmail.com
Purpose: Pseudomonas aeruginosa is associated with pulmonary function decline and high disease severity in non-cystic fibrosis (CF) bronchiectasis. The prevalence of nontuberculous mycobacteria (NTM) in non-CF bronchiectasis patients has increased recently. This study investigated the impact of NTM with or without $P$. aeruginosa isolates in non-CF bronchiectasis patients. Patients and methods: Our retrospective study included 96 non-CF bronchiectasis patients from January 2005 to December 2014. We recorded the presentation, exacerbations, emergency department (ED) visits, hospitalization, serial pulmonary function, radiologic studies, and sputum culture results. All patients were followed up for at least 2 years.

Results: The 96 patients were divided into four groups: patients with concomitant negative NTM and $P$. aeruginosa isolates ( $\mathrm{n}=41$; group 1$)$, patients with positive NTM isolates $(\mathrm{n}=20$; group 2), patients with positive $P$. aeruginosa isolates ( $\mathrm{n}=20$; group 3 ), and patients with concomitant positive NTM and $P$. aeruginosa isolates ( $\mathrm{n}=15$; group 4). Compared with group 1 patients, patients in groups 2 and 3 showed a significant decline in forced expiratory volume in 1 second $\left(\mathrm{FEV}_{1}\right)$. They also had more frequent annual acute exacerbations (AE), ED visits, and hospitalization. Group 4 patients had the greatest $\mathrm{FEV}_{1}$ and forced vital capacity (FVC) decline and the most frequent $\mathrm{AE}, \mathrm{ED}$ visits, and hospitalization.

Conclusion: Concomitant NTM and P. aeruginosa isolates in non-CF bronchiectasis are associated with the greatest pulmonary function decline and the worst disease severity. This result suggested that early recognition and prompt treatment of concomitant NTM and P. aeruginosa isolates may improve the outcome in non-CF bronchiectasis patients.

Keywords: nontuberculous mycobacteria, Pseudomonas aeruginosa, non-cystic fibrosis bronchiectasis

\section{Introduction}

Non-cystic fibrosis (CF) bronchiectasis is a chronic, progressive inflammatory lung disease characterized by irreversible dilatation of the bronchi and recurrent infections. ${ }^{1,2}$ Two prognostic indices that aid clinical decisions are the bronchiectasis severity index (BSI) and the FACED score. FACED stands for forced expiratory volume in 1 second $\left(\mathrm{FEV}_{1}\right)$, age, chronic colonization of Pseudomonas aeruginosa, radiological extension, and dyspnea. ${ }^{3,4}$ The BSI was developed to predict mortality, exacerbations, and the quality of life of bronchiectasis patients, whereas the FACED score specifically focuses on predicting mortality. ${ }^{4,5}$ However, the primary issues for non-CF bronchiectasis patients include not only exacerbations and mortality but also pulmonary function decline, emergency department (ED) visits, hospitalization, and medical costs. ${ }^{6}$ 
P. aeruginosa is a well-documented pathogen that provokes an intense inflammatory response leading to persistent airway inflammation and airway structural damage. ${ }^{7}$ Bronchiectasis along with chronic colonization by $P$. aeruginosa is also associated with greater pulmonary function decline, more frequent exacerbations and hospitalization, and mortality. ${ }^{8-10}$ Hence, chronic colonization by $P$. aeruginosa is an important component of both the BSI and the FACED score.

Bronchiectasis and nontuberculous mycobacteria (NTM) are interrelated, but which one first affects patients is not known. ${ }^{11}$ The prevalence of NTM in bronchiectasis ranges widely $(2 \%-37 \%)$ and has increased in recent years, probably due to improvements in diagnostic techniques and the increasing attention given to the pathogen. ${ }^{11-15}$ Elderly women with a low body mass index (BMI) are more susceptible to NTM infection in non-CF bronchiectasis, ${ }^{15}$ nevertheless, the clinical implication of NTM in non-CF bronchiectasis is still an unresolved question. In addition, with regard to compliance, patients' preference, and undetermined benefits, therapy initiation has been controversial and not mandatory. ${ }^{16}$

In this study, we investigated the implications of NTM and Pseudomonas isolates for non-CF bronchiectasis patients. This approach may provide us with more information that will aid clinical decision-making.

\section{Patients and methods}

\section{Patients}

In our retrospective study, we included 96 non-CF bronchiectasis adult patients admitted to Linkou Medical Center, Chang Gung Memorial Hospital, Taiwan, from January 2005 to December 2014. The study was approved by the institutional review board of Chang Gung Memorial Hospital (approval no. 97-1105A3). Informed consent was waived because this was a retrospective study and there was no modification in patient management. All personal information was encrypted in the database, and patient data accessed was de-identified. There was no breach of privacy. All patients underwent high-resolution computed tomography, confirming bronchiectasis. No patient had a background suggesting CF, such as chronic pancreatic, liver, or intestinal dysfunction, electrolyte imbalance, disease onset before adolescence, or a family history of CF.

All the patients were followed up for at least 2 years. Follow-up information was obtained from the patients' medical records. Data related to the patients' gender, age, smoking status, BMI, BSI, at least two pulmonary function tests during follow-up, and mortality were collected. We defined acute exacerbations (AE) as an event that was clinically diagnosed by the physician and required antibiotic prescription for acute onset of increasing cough, worsening dyspnea, and changes in sputum characteristics (eg, volume, consistency, and purulence). We also recorded the frequency of annual AE, as well as ED visits and hospitalization due to bronchiectasis AE.

For every patient, we cultured at least two sputum samples for Pseudomonas and three sputum samples were cultured for mycobacteria in the stable state. Patients who had had pulmonary tuberculosis before, had undergone anti-NTM therapy, had incomplete medical records, and missed their follow-up were excluded.

On the basis of the pathogens isolated from their sputum cultures, the patients were divided into four groups: group 1, concomitant negative NTM and $P$. aeruginosa isolates; group 2, positive NTM isolates; group 3, positive $P$. aeruginosa isolates; and group 4, concomitant positive NTM and $P$. aeruginosa isolates.

\section{Pulmonary function}

Spirometry was performed as per recommendations made by the American Thoracic Society/European Respiratory Society. ${ }^{17}$ Pulmonary function data recorded nearest the firsttime NTM or P. aeruginosa isolate in groups 2-4 and nearest the time the first sputum sample was sent for mycobacterial culture in group 1 were compared with those most recently recorded in the specific group.

\section{Statistical analysis}

We used Fisher's exact test to compare categorical variables and presented them as frequencies (percentages). We then compared intergroup pulmonary function data using the unpaired Student's $t$-test, and follow-up pulmonary function data were compared using the paired Student's $t$-test. All analyses were two-sided, and $p<0.05$ was considered statistically significant. Statistical analyses were performed using Prism version 5 (GraphPad Software Inc., La Jolla, CA, USA) and SPSS Statistics version 20.0 (IBM Corporation, Armonk, NY, USA).

\section{Results}

\section{Patient characteristics}

Data from all 96 adult patients diagnosed with non-CF bronchiectasis were analyzed. Of the 96 patients, 41 were in group 1,20 in group 2, 20 in group 3, and 15 in group 4. No patient received anti-NTM therapy. Table 1 summarizes the clinical characteristics of all patients. The mean age of the patients was $65.3 \pm 13.1$ years. Age, gender, smoking status, and the BMI were not significantly different between the four groups. Compared to group 1, groups $2-4$ had a significantly greater BSI ( $p=0.0176,<0.0001$, and $<0.0001$, respectively; Table 1). 


\section{Exacerbations}

The median follow-up duration was 2.79 years (range 2.13-10.7 years). Compared to group 1, group 2 patients had frequent annual $\mathrm{AE}$ (1.21 \pm 1.63 vs $2.70 \pm 2.48 ; p=0.0083)$, more ED visits $(0.15 \pm 0.38$ vs $0.72 \pm 0.95 ; p=0.024)$, and more hospitalization $(0.03 \pm 0.17$ vs $0.45 \pm 0.77 ; p=0.0019$; Table 1 and Figure 1). The annual AE, ED visits, and hospitalization were not significantly different between groups 2 and 3 (Table 1). Group 4 had the most frequent annual $\mathrm{AE}$ (6.92 \pm 6.47 , $p<0.0001)$, ED visits $(0.74 \pm 1.04, p=0.00394)$, and hospitalization (1.28 $\pm 2.16, p=0.0005)$ (Table 1 and Figure 1). During follow-up, there were only nine deaths and the mortality rate was not different between the four groups (Table 1).

\section{Pulmonary function}

The baseline $\mathrm{FEV}_{1}$ and forced vital capacity (FVC) were similar between groups 1 and 2 ( $p=0.496$ and 0.988 , respectively; Table 2). Groups 2 and 4 had significantly worse baseline $\mathrm{FEV}_{1}$ ( $p=0.0002$ and 0.0167 , respectively) and FVC ( $p=0.0006$ and 0.016 , respectively) compared with group 1 (Table 2). Groups 2-4 showed a significant decline in $\mathrm{FEV}_{1}$ (Table 2 and Figure 2). There was no significant change in the baseline and follow-up $\mathrm{FEV}_{1}$ values in group 1 (Table 2 and Figure 2). The FVC decline was significant in group 4 and was borderline statistically significant in group 3 (Table 2 and Figure 3), but serial FVC values were not significantly different in groups 1 and 2 (Table 2 and Figure 3).

\section{Mycobacterial species}

Of the 96 patients, sputum cultures of 35 patients showed positive NTM isolates: 11 patients had multiple NTM isolates, whereas the remaining 24 patients had a single NTM isolate. The Mycobacterium avium-M ycobacterium intracellulare complex was the most common NTM species found (18 of

Table I Clinical characteristics of non-CF bronchiectasis patients in the four groups according to the pathogen isolated from sputum samples

\begin{tabular}{|c|c|c|c|c|c|}
\hline Characteristics & $\begin{array}{l}\text { Group I } \\
n=4 I\end{array}$ & $\begin{array}{l}\text { Group } 2 \\
\mathrm{n}=\mathbf{2 0}\end{array}$ & $\begin{array}{l}\text { Group } 3 \\
n=20\end{array}$ & $\begin{array}{l}\text { Group } 4 \\
n=15\end{array}$ & $p$-value \\
\hline Age, mean $\pm S D$ & $64.0 \pm 13.7$ & $63.7 \pm 12.2$ & $67.0 \pm 14.0$ & $68.8 \pm 11.5$ & 0.562 \\
\hline Female, n (\%) & $16(39.0)$ & II (55) & $13(65)$ & II (73.3) & 0.075 \\
\hline Smoking, n (\%) & $6(14.6)$ & $5(25)$ & $5(25)$ & $3(20)$ & 0.712 \\
\hline $\mathrm{BMI}, \mathrm{kg} / \mathrm{m}^{2}$, mean $\pm \mathrm{SD}$ & $22.5 \pm 3.2$ & $21.3 \pm 2.9$ & $20.7 \pm 3.1$ & $22.5 \pm 3.5$ & 0.1501 \\
\hline BSI score, mean \pm SD & $4.91 \pm 3.12$ & $7.56 \pm 4.80$ & $10.67 \pm 4.74$ & II. $.4 \pm 4.52$ & $<0.0001$ \\
\hline $\mathrm{AE}$, annual, mean $\pm \mathrm{SD}$ & $1.21 \pm 1.63$ & $2.70 \pm 2.48$ & $1.94 \pm 1.78$ & $6.92 \pm 6.47$ & $<0.000$ I \\
\hline ED visit, annual, mean $\pm S D$ & $0.15 \pm 0.38$ & $0.72 \pm 0.95$ & $0.7 \mathrm{I} \pm \mathrm{I} .44$ & $0.74 \pm 1.04$ & 0.00394 \\
\hline Hospitalization, annual, mean \pm SD & $0.03 \pm 0.17$ & $0.45 \pm 0.77$ & $0.16 \pm 0.34$ & $1.28 \pm 2.16$ & 0.0005 \\
\hline Mortality, n (\%) & $2(4.9)$ & $2(10)$ & $3(15)$ & $2(13.3)$ & 0.5711 \\
\hline
\end{tabular}

Notes: Group I, concomitant negative NTM and Pseudomonas aeruginosa isolates; group 2, positive NTM isolates; group 3, positive $P$. aeruginosa isolates; group 4, concomitant positive NTM and $P$. aeruginosa isolates.

Abbreviations: AE, acute exacerbations; BMI, body mass index; BSI, bronchiectasis severity index; CF, cystic fibrosis; ED, emergency department; NTM, nontuberculous mycobacteria; SD, standard deviation.
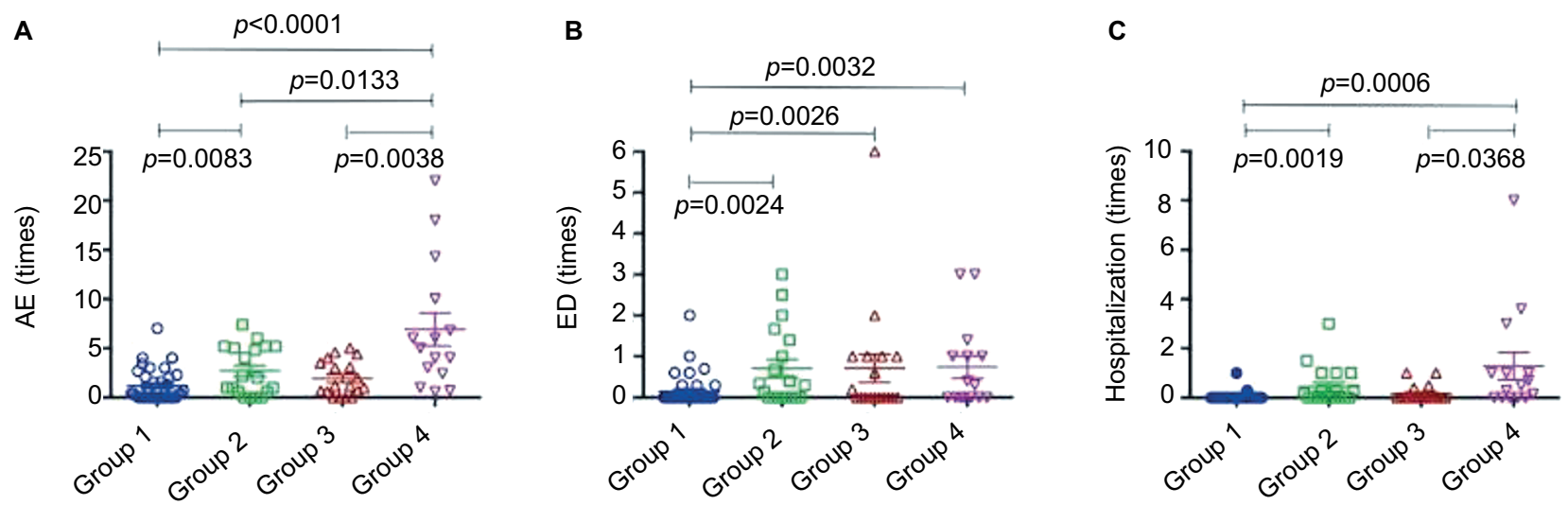

Figure I Comparison of clinical disease severity in patients with non-CF bronchiectasis in the four groups according to the pathogen isolated from sputum samples. Notes: Group I, concomitant negative NTM and Pseudomonas aeruginosa isolates; group 2, positive NTM isolates; group 3, positive P. aeruginosa isolates; group 4, concomitant positive NTM and P. aeruginosa isolates. (A) Annual acute exacerbations; (B) emergency department visits; (C) hospitalization. Abbreviations: AE, acute exacerbations; CF, cystic fibrosis; ED, emergency department; NTM, nontuberculous mycobacteria. 
Table 2 Comparison of baseline and follow-up pulmonary function in patients with non-CF bronchiectasis in the four groups according to the pathogen isolated from sputum samples

\begin{tabular}{|c|c|c|c|c|c|}
\hline & $\begin{array}{l}\text { Group I } \\
n=41\end{array}$ & $\begin{array}{l}\text { Group } 2 \\
n=20\end{array}$ & $\begin{array}{l}\text { Group } 3 \\
n=20\end{array}$ & $\begin{array}{l}\text { Group } 4 \\
n=15\end{array}$ & $p$-value \\
\hline Baseline $\mathrm{FEV}_{1}, \mathrm{~L}$, mean $\pm \mathrm{SD}$ & $1.62 \pm 0.60$ & $1.76 \pm 0.97$ & $1.02 \pm 0.38$ & $1.19 \pm 0.49$ & 0.0008 \\
\hline Follow-up $\mathrm{FEV}_{1}, \mathrm{~L}$, mean $\pm \mathrm{SD}$ & $1.57 \pm 0.59$ & $1.63 \pm 0.88$ & $0.89 \pm 0.35$ & $1.03 \pm 0.42$ & $<0.0001$ \\
\hline Decrease in $\mathrm{FEV}_{1}, \mathrm{~L}$, mean $\pm \mathrm{SD}$ & $\begin{array}{l}-0.04 \pm 0.21 \\
p=0.204\end{array}$ & $\begin{array}{l}-0.13 \pm 0.28 \\
p=0.049 *\end{array}$ & $\begin{array}{l}-0.13 \pm 0.26 \\
p=0.04 I^{*}\end{array}$ & $\begin{array}{l}-0.16 \pm 0.27 \\
p=0.038^{*}\end{array}$ & \\
\hline Baseline FVC, L, mean \pm SD & $2.15 \pm 0.68$ & $2.16 \pm 1.13$ & $|.53 \pm 0.5|$ & $1.67 \pm 0.52$ & 0.0068 \\
\hline Follow-up FVC, L, mean \pm SD & $2.14 \pm 0.65$ & $2.10 \pm 1.00$ & $1.38 \pm 0.58$ & $1.39 \pm 0.48$ & $<0.0001$ \\
\hline Decrease in FVC, L, mean \pm SD & $\begin{array}{l}-0.01 \pm 0.28 \\
p=0.818\end{array}$ & $\begin{array}{l}-0.06 \pm 0.32 \\
p=0.437 * *\end{array}$ & $\begin{array}{l}-0.15 \pm 0.33 \\
p=0.056 * *\end{array}$ & $\begin{array}{l}-0.28 \pm 0.33 \\
p=0.0052^{* *}\end{array}$ & \\
\hline
\end{tabular}

Notes: Group I, concomitant negative NTM and Pseudomonas aeruginosa isolates; group 2, positive NTM isolates; group 3, positive P. aeruginosa isolates; group 4, concomitant positive NTM and P. aeruginosa isolates. *Follow-up FEV, compared to baseline FEV. ***Follow-up FVC compared to baseline FVC.

Abbreviations: CF, cystic fibrosis; FEV , forced expiratory volume in I second; FVC, forced vital capacity; NTM, nontuberculous mycobacteria; SD, standard deviation.

A

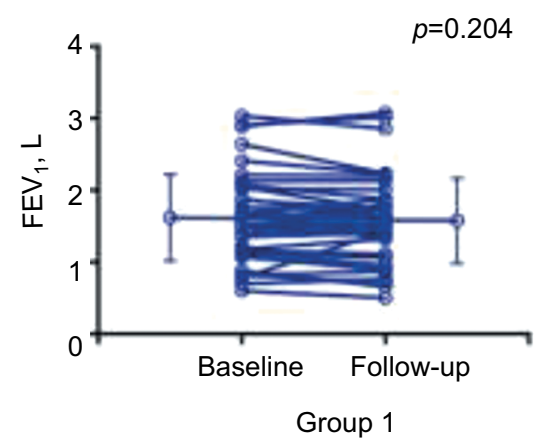

C

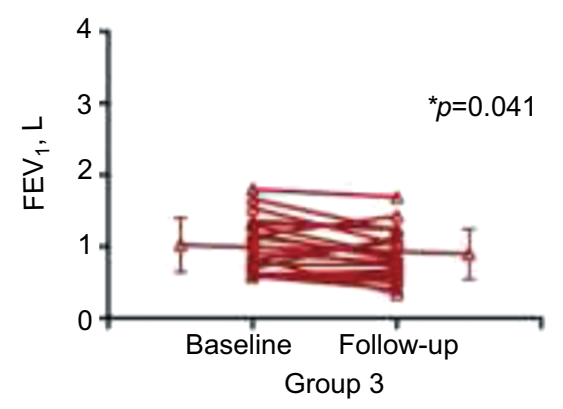

B
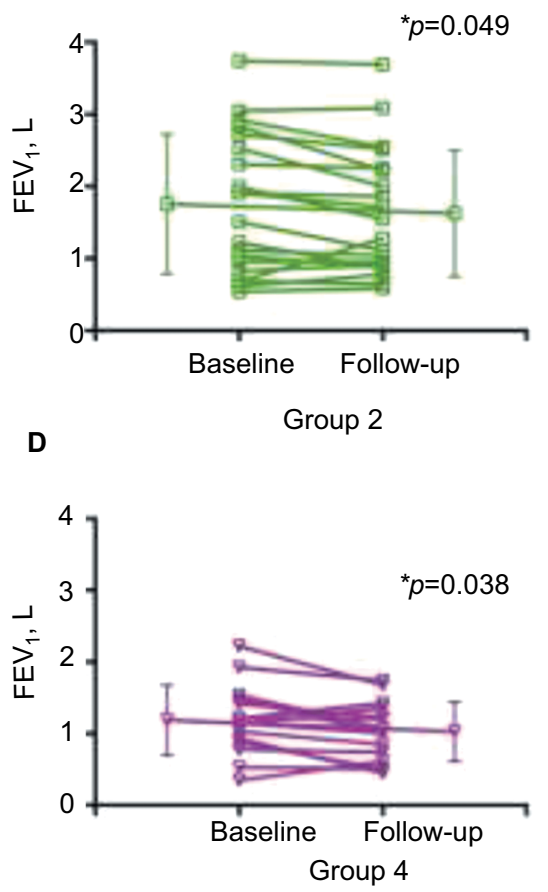

Figure 2 Comparison of FEV, decline in patient with non-CF bronchiectasis in the four groups according to the pathogen isolated from sputum samples.

Notes: (A) Group I, concomitant negative NTM and Pseudomonas aeruginosa isolates, mean FEV, decline: $-0.04 \pm 0.2$ I; (B) group 2, positive NTM isolates, mean FEV decline: $-0.13 \pm 0.28$; (C) group 3, positive $P$. aeruginosa isolates, mean FEV, decline: $-0.13 \pm 0.26$; (D) group 4 , concomitant positive NTM and $P$. aeruginosa isolates, mean FEV, decline: $-0.16 \pm 0.27$. * Statistical significance.

Abbreviations: CF, cystic fibrosis; $\mathrm{FEV}_{1}$, forced expiratory volume in I second; NTM, nontuberculous mycobacteria.

35 patients, $51.4 \%$ ), followed by Mycobacterium fortuitum and Mycobacterium chelonae (eight of 35 patients, $22.9 \%$ ). There were six patients without an identified NTM species. The NTM species were not significantly different between groups 2 and 4 (Table 3).

\section{Discussion}

To the best of our knowledge, this is the first study to evaluate the clinical implications of NTM with or without
Pseudomonas isolates in non-CF bronchiectasis patients. In our 96 patients with a median follow-up duration of 2.79 years, patients with positive NTM isolates (group 2) and positive $P$. aeruginosa isolates (group 3) showed a high decline in their $\mathrm{FEV}_{1}$ and frequent annual $\mathrm{AE}$, ED visits, and hospitalization. Patients with concomitant positive NTM and P. aeruginosa isolates (group 4) showed the greatest decline in their $\mathrm{FEV}_{1}$ and $\mathrm{FVC}$ and the most frequent annual $\mathrm{AE}, \mathrm{ED}$ visits, and hospitalization. 
A

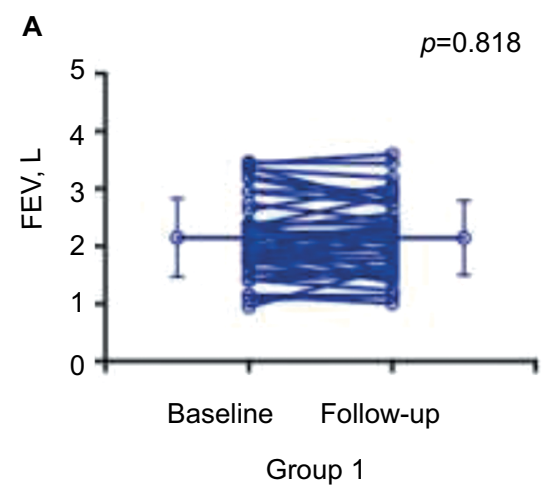

C

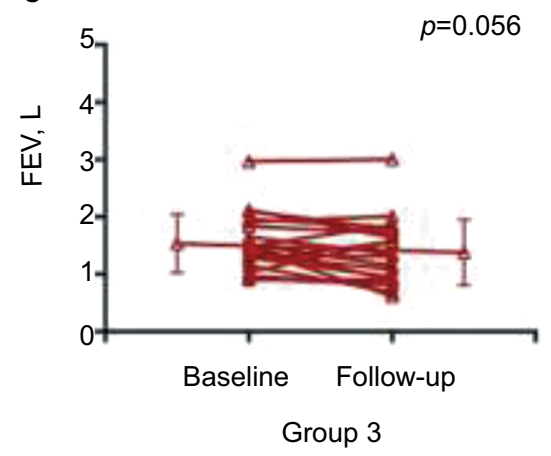

B

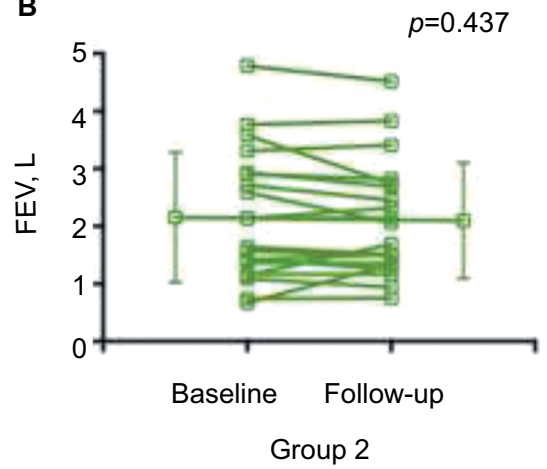

D

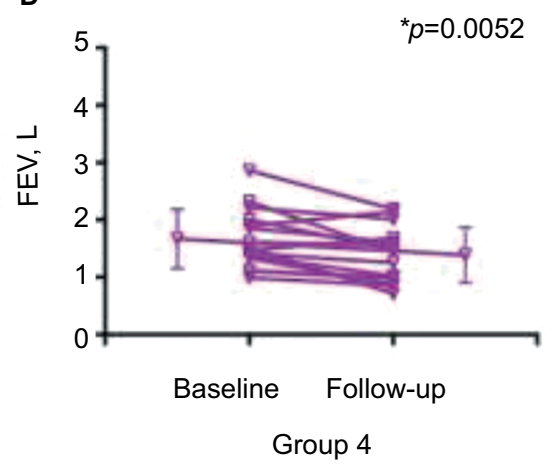

Figure 3 Comparison of FVC decline in patients with non-CF bronchiectasis in the four groups according to the pathogen isolated from sputum samples.

Notes: (A) Group I, concomitant negative NTM and Pseudomonas aeruginosa isolates, mean FEV, decline: -0.0 I \pm 0.28 ; (B) group 2, positive NTM isolates, mean FEV decline: $-0.06 \pm 0.32$; (C) group 3, positive Pseudomonas aeruginosa isolates, mean FEV, decline: $-0.15 \pm 0.33$; (D) group 4, concomitant positive NTM and $P$. aeruginosa isolates, mean FEV decline: $-0.28 \pm 0.33$. *Statistical significance.

Abbreviations: CF, cystic fibrosis; FEVI, forced expiratory volume in I second; FVC, forced vital capacity; NTM, nontuberculous mycobacteria.

Table 3 NTM species isolated from sputum samples in non-CF bronchiectasis patients

\begin{tabular}{|c|c|c|}
\hline NTM species & $\begin{array}{l}\text { Group } 2 \\
n=20 \\
n(\%)\end{array}$ & $\begin{array}{l}\text { Group } 4 \\
n=15 \\
n(\%)\end{array}$ \\
\hline $\begin{array}{l}\text { Mycobacterium avium-Mycobacterium } \\
\text { intracellulare complex }\end{array}$ & $10(50)$ & $8(53.3)$ \\
\hline Mycobacterium fortuitum & $2(10)$ & $6(40)$ \\
\hline Mycobacterium chelonae & $4(20)$ & $4(26.7)$ \\
\hline Mycobacterium abscessus & $2(10)$ & $3(20)$ \\
\hline Mycobacterium gordonae & $2(10)$ & $2(13.3)$ \\
\hline Mycobacterium kansasii & I (5) & $0(0)$ \\
\hline Mycobacterium mageritense & I (5) & $0(0)$ \\
\hline Mycobacterium scrofulaceum & $0(0)$ & I (6.7) \\
\hline Mycobacterium peregrinum & $0(0)$ & I (6.7) \\
\hline Unidentified & $4(20)$ & $2(13.3)$ \\
\hline
\end{tabular}

Abbreviations: CF, cystic fibrosis; NTM, nontuberculous mycobacteria.

The BSI and the FACED score are two well-known prognostic indices for bronchiectasis. ${ }^{3,4}$ The BSI accurately predicts mortality, exacerbations, hospitalization, quality of life, respiratory symptoms, 6 min walk distance, and pulmonary function decline. BSI-predicted outcomes may be the real problems that need to be resolved in clinical practice. ${ }^{6}$ Chronic Pseudomonas colonization in bronchiectasis patients represents higher disease severity and risk of exacerbations. ${ }^{10}$ It is also a common factor in the BSI and the FACED score. However, the severity implication of NTM isolates remains unknown.

In our study, we observed that group 2 had a higher BSI. Although the BSI increased in group 3, it was the highest in group 4. We think that along with a higher BSI, NTM isolates from the sputum may also play a role in clinical disease severity in non-CF bronchiectasis patients. In one prospective, observational study, Faverio et al showed that patients with pulmonary NTM infection have lower disease severity, BSI, and exacerbations compared with patients with chronic Pseudomonas infection. ${ }^{18}$ However, they did not analyze patients with concomitant positive NTM and $P$. aeruginosa isolates. In our study, we divided patients into four groups and showed that patients with positive NTM isolates (group 2) had more frequent annual AE, ED visits, and hospitalization compared with patients with concomitant negative NTM and P. aeruginosa isolates (group 1). Also, patients with positive P. aeruginosa isolates (group 3) showed similar data as group 2 patients. However, patients with concomitant positive NTM and P. aeruginosa isolates (group 4) had the most frequent annual AE, ED visits, and 
hospitalization. Disease severity seemed to rise in groups 2 and 3 and was most severe in group 4.

Huang et al found that patients with NTM isolates and chronic obstructive pulmonary disease (COPD) have more frequent AEs and that multiple NTM isolates are associated with a rapid decline of pulmonary function in COPD patients. ${ }^{19}$ Pseudomonas colonization, frequent AE, and systemic inflammation have been known to be associated with a rapid decline of pulmonary function in non-CF bronchiectasis patients $;^{8}$ however, the impact of NTM on pulmonary function in non-CF bronchiectasis remains unknown. In our study, we observed that group 2 patients had similar baseline $\mathrm{FEV}_{1}$ and $\mathrm{FVC}$ values as did group 1 patients and that, comparatively, group 3 patients had worse baseline $\mathrm{FEV}_{1}$ and FVC values. However, the $\mathrm{FEV}_{1}$ decline was significant in groups 2 and 3. Moreover, in group 4 patients, both $\mathrm{FEV}_{1}$ and FVC deteriorated most significantly, suggesting that not only Pseudomonas but also NTM is associated with pulmonary function decline.

With regard to anti-NTM therapy, in M. avium complex (MAC) lung disease, Khan et al observed improvement in pulmonary function after patients were administered antiMAC therapy. ${ }^{20}$ In one prospective NTM registry cohort in Korea, Park et al found that treatment failure in NTM lung disease is associated with a greater decline in pulmonary function. ${ }^{21}$ However, the decline in $\mathrm{FEV}_{1}$ and FVC was similar to that in patients with successful treatment. On the contrary, Mehta et al demonstrated nonsignificant changes in pulmonary function of patients after treatment for NTM lung disease. ${ }^{22}$ In addition to the undetermined benefits of antiNTM therapy, the major problems include a long treatment period, patients' compliance, disease severity, and possible adverse effects of the medication. Therefore, in our study, we excluded patients who had received anti-NTM therapy, and we presented the natural course of NTM isolation in non-CF bronchiectasis patients.

Since NTM may play a prognostic role in disease severity and pulmonary function decline, it is important to identify the risk factors of NTM isolation in bronchiectasis. Mirsaeidi et al found that elderly women with a low BMI were at high risk for NTM infection in non-CF bronchiectasis. ${ }^{15}$ Maiz et al showed that an FVC $>75 \%$, age $>50$ years, and a BMI $<23 \mathrm{~kg} / \mathrm{m}^{2}$ are independently associated with the presence of NTM in non-CF bronchiectasis patients. ${ }^{23}$ In our study, we did not find any correlations of age, gender, smoking status, and the BMI with positive NTM isolation, probably due to the small sample population.
Our study had several inherent limitations. First, it was a retrospective study and we excluded patients with previous pulmonary tuberculosis and those who had received anti-NTM therapy; this probably led to selection biases and diminished generalizability. Second, the spirometry interval was inconsistent. However, the follow-up period was about 3 years and was similar between groups, so the pulmonary function decline may still provide useful information in a clinical setting. Third, because of the small number of deaths in our sample population, we could not analyze factors related to mortality. Owing to the retrospective nature of our data, the sample size was limited. Larger, prospective studies are required.

\section{Conclusion}

Our study showed that NTM isolates from the sputum of non$\mathrm{CF}$ bronchiectasis patients are associated with greater $\mathrm{FEV}_{1}$ decline and AE. Patients with concomitant positive NTM and $P$. aeruginosa isolates have the greatest pulmonary function decline and the most frequent AE. These findings suggest that NTM may play an important role in disease severity and pulmonary function decline in non-CF bronchiectasis patients. Further prospective, large-scale, multicenter studies are warranted to validate our results and identify the risk factors of NTM isolation in non-CF bronchiectasis.

\section{Availability of data and materials}

The data sets analyzed during the current study are available from the corresponding author upon reasonable request.

\section{Acknowledgments}

We thank all the investigators and members of the Department of Thoracic Care Medicine for their efforts. This research did not receive any specific grant from funding agencies in the public, commercial, or not-for-profit sectors.

\section{Author contributions}

All the authors contributed to conception and design of the study. MHH, CYL, CYW, and HCL analyzed and interpreted the data. MHH, CYL, YLL, and SML drafted the manuscript. MHH, SML, and HCL provided the study materials and selected patients. CYL, CYW, and YFF collected and assembled data. All the authors draft the article or revising it critically for important intellectual content, approved the final manuscript and agreed to be accountable for all aspects of the work in ensuring that questions related to the accuracy or integrity of any part of the work are appropriately investigated and resolved. 


\section{Disclosure}

The authors report no conflicts of interest in this work.

\section{References}

1. Hsieh MH, Chou PC, Chou CL, et al. Matrix metalloproteinase-1 polymorphism $(-1607 \mathrm{G})$ and disease severity in non-cystic fibrosis bronchiectasis in Taiwan. PLoS One. 2013;8(6):e66265.

2. Hsieh MH, Fang YF, Chen GY, et al. The role of the high-sensitivity $\mathrm{C}$-reactive protein in patients with stable non-cystic fibrosis bronchiectasis. Pulm Med. 2013;2013:795140.

3. Martinez-Garcia MA, de Gracia J, Vendrell Relat M, et al. Multidimensional approach to non-cystic fibrosis bronchiectasis: the FACED score. Eur Respir J. 2014;43(5):1357-1367.

4. Chalmers JD, Goeminne P, Aliberti S, et al. The bronchiectasis severity index. An international derivation and validation study. Am J Respir Crit Care Med. 2014;189(5):576-585.

5. Ellis HC, Cowman S, Fernandes M, Wilson R, Loebinger MR. Predicting mortality in bronchiectasis using bronchiectasis severity index and FACED scores: a 19-year cohort study. Eur Respir J. 2016;47(2):482-489.

6. McDonnell MJ, Aliberti S, Goeminne PC, et al. Multidimensional severity assessment in bronchiectasis: an analysis of seven European cohorts. Thorax. 2016;71(12):1110-1118.

7. Rogers GB, Zain NM, Bruce KD, et al. A novel microbiota stratification system predicts future exacerbations in bronchiectasis. Ann Am Thorac Soc. 2014;11(4):496-503.

8. Martinez-Garcia MA, Soler-Cataluna JJ, Perpina-Tordera M, RomanSanchez P, Soriano J. Factors associated with lung function decline in adult patients with stable non-cystic fibrosis bronchiectasis. Chest. 2007;132(5):1565-1572.

9. McDonnell MJ, Jary HR, Perry A, et al. Non cystic fibrosis bronchiectasis: a longitudinal retrospective observational cohort study of Pseudomonas persistence and resistance. Respir Med. 2015;109(6):716-726.

10. Finch S, McDonnell MJ, Abo-Leyah H, Aliberti S, Chalmers JD. A comprehensive analysis of the impact of Pseudomonas aeruginosa colonization on prognosis in adult bronchiectasis. Ann Am Thorac Soc. 2015;12(11):1602-1611.
11. Bonaiti G, Pesci A, Marruchella A, Lapadula G, Gori A, Aliberti S. Nontuberculous mycobacteria in noncystic fibrosis bronchiectasis. Biomed Res Int. 2015;2015:197950.

12. Wickremasinghe M, Ozerovitch LJ, Davies G, et al. Non-tuberculous mycobacteria in patients with bronchiectasis. Thorax. 2005;60(12):1045-1051

13. Fowler SJ, French J, Screaton NJ, et al. Nontuberculous mycobacteria in bronchiectasis: prevalence and patient characteristics. Eur Respir J. 2006;28(6):1204-1210.

14. Chu H, Zhao L, Xiao H, et al. Prevalence of nontuberculous mycobacteria in patients with bronchiectasis: a meta-analysis. Arch Med Sci. 2014;10(4):661-668.

15. Mirsaeidi M, Hadid W, Ericsoussi B, Rodgers D, Sadikot RT. Nontuberculous mycobacterial disease is common in patients with non-cystic fibrosis bronchiectasis. Int J Infect Dis. 2013;17(11):e1000-e1004.

16. Griffith DE, Aksamit T, Brown-Elliott BA, et al. An official ATS/IDSA statement: diagnosis, treatment, and prevention of nontuberculous mycobacterial diseases. Am J Respir Crit Care Med. 2007;175(4):367-416.

17. Miller MR, Hankinson J, Brusasco V, et al. Standardisation of spirometry. Eur Respir J. 2005;26(2):319-338.

18. Faverio P, Stainer A, Bonaiti G, et al. Characterizing non-tuberculous mycobacteria infection in bronchiectasis. Int J Mol Sci. 2016;17(11):E1913.

19. Huang CT, Tsai YJ, Wu HD, et al. Impact of non-tuberculous mycobacteria on pulmonary function decline in chronic obstructive pulmonary disease. Int J Tuberc Lung Dis. 2012;16(4):539-545.

20. Khan Z, Miller A, Bachan M, Donath J. Mycobacterium avium complex (MAC) lung disease in two inner city community hospitals: recognition, prevalence, co-infection with mycobacterium tuberculosis (MTB) and pulmonary function (PF) improvements after treatment. Open Respir Med J. 2010;4:76-81.

21. Park HY, Jeong BH, Chon HR, Jeon K, Daley CL, Koh WJ. Lung function decline according to clinical course in nontuberculous mycobacterial lung disease. Chest. 2016;150(6):1222-1232.

22. Mehta M, Chapman KR, Heffer M, Marras TK. Impact of pulmonary nontuberculous mycobacterial treatment on pulmonary function tests in patients with and without established obstructive lung disease. Respirology. 2015;20(6):987-993.

23. Maiz L, Giron R, Olveira C, Vendrell M, Nieto R, Martinez-Garcia MA. Prevalence and factors associated with nontuberculous mycobacteria in non-cystic fibrosis bronchiectasis: a multicenter observational study. BMC Infect Dis. 2016;16(1):437.
Infection and Drug Resistance

\section{Publish your work in this journal}

Infection and Drug Resistance is an international, peer-reviewed openaccess journal that focuses on the optimal treatment of infection (bacterial, fungal and viral) and the development and institution of preventive strategies to minimize the development and spread of resistance. The journal is specifically concerned with the epidemiology of antibiotic

\section{Dovepress}

resistance and the mechanisms of resistance development and diffusion in both hospitals and the community. The manuscript management system is completely online and includes a very quick and fair peerreview system, which is all easy to use. Visit http://www.dovepress.com/ testimonials.php to read real quotes from published authors. 